\title{
Les interférences occlusales sont-elles pathogènes ?
}

\section{Occlusal interference are they pathogenic?}

\author{
Résumé
}

MOTS-CLEFS :
- Dysfonctionnement,
tests cliniques,
examen clinique
KEYWORDS:
- Dysfunction,
clinical Tests,
clinical examination

$\operatorname{AOS} n^{\circ} 290-2018$
Les interférences occlusales ont été longtemps considérées comme un facteur étiologique des troubles temporo- mandibulaires. Il est actuellement établi que celles-ci ne prennent qu'une très faible part dans l'apparition de ces dysfonctionnements musculosquelettiques. Ainsi les interférences occlusales sont considérées comme une adaptation de l'appareil manducateur et non responsables des troubles temporo-mandibulaires.

En outre, les interférences occlusales augmentent les difficultés d'accomplissement des fonctions d'incision et de phonation. La prise de décision d'aménagement occlusal est très controversée et actuellement limitée aux traitements de dentisterie restauratrice.

\author{
Abstract
}

The role of occlusal interference as an etiologic factor of TMD dysfunction has been discussed for a long time. Now, it has been well established that occlusal interferences play a very little part in this dysfunction. Therefore, occlusal interferences are considered as an adaptation and not the cause for the disturbance of manducatory functions Furthermore, occlusal interferences exacerbate difficulties in performing incision and phonation.

Decision making in occlusal adjusment is very controversial and indication are now limited to oral rehabilitation and restorative dentistry.

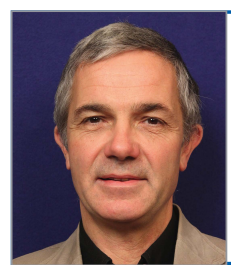

- Bernard FLEITER, Maître de Conférences des Universités - Praticien Hospitalier, Faculté de Chirurgie Dentaire, Université Paris René Descartes. Responsable de la consultation de prise en charge de la douleur et des troubles fonctionnels orofaciaux. Hôpital Charles Foix, Ivry-sur-Seine-bfleiter@wanadoo.fr

\section{INTRODUCTION}

Beaucoup de praticiens ont appris à repérer et parfois à détester les interférences occlusales. Qu'en est-il vraiment ? Faut-il les éliminer et comment ? Cette démarche ne constitue-t-elle pas une démarche excessive, de nature à être assimilée à un surtraitement dans certains cas iatrogène?

\section{DÉFINITION}

Une interférence occlusale peut être considérée comme un obstacle au déplacement aisé de la mandibule au cours des déplacements mandibulaires, pouvant nuire au déroulement de certaines fonctions manducatrices comme la mastication, la dilacération la déglutition, et surtout l'incision. Dans ces conditions certains contacts dentaires, lors des mouvements de propulsion et de diduction, seraient limités voire totalement empêchés.

Lors de la propulsion on observera si le guidage est bien effectué par l'affrontement des incisives, canines et premières prémolaires mandibulaires sur les six dents antérieures maxillaires. Dans certains cas d'autres dents peuvent participer au guidage sans dommage particulier. Dans d'autres cas, à l'inverse, une ou plusieurs dents postérieures peuvent interdire le contact et donc le guidage par les dents antérieures en propulsion. Nous parlerons alors d'interférences 
occlusales non travaillantes en propulsion. Il est fréquent dans ce cas que la fonction d'incision soit perturbée voire impossible.

Si l'on examine les déplacements mandibulaires lors d'un déplacement vers la droite, le guidage s'effectue le plus souvent par des contacts continus sur la canine et incisive latérale maxillaire (Fig. 1a, b), parfois les prémolaires (Fig. 2a, b, c), voire les molaires du côté droit, appelé coté mastiquant ou travaillant. Il peut arriver qu'un autre contact soit observé du côté gauche, sans perturber le déplacement et sans faire perdre les contacts guidants du côté travaillant. Ceci ne constitue pas pour autant une interférence non travaillante, mais une occlusion dite balancée que l'on retrouve chez beaucoup de patients ne présentant par ailleurs aucun signe ou symptôme de Dysfonctionnement de l'Appareil Manducateur (DAM), ni gêne lors de l'établissement des fonctions. C'est précisément l'occlusion recherchée lors de la réalisation d'une prothèse complète bimaxillaire dans le but de stabiliser lesdites prothèses lors des excursions mandibulaires en contact occlusaux et surtout lors de la mastication. Néanmoins, si ce ou ces contacts observés du côté gauche lors d'une diduction droite sont les seuls à s'établir, c'est-à-dire que les contacts guidants du côté travaillant ou mastiquant ne s'exercent plus, nous sommes en présence d'une interférence occlusale non travaillante. Ainsi, on observera la continuité de la trace de guidage du côté droit. Si celle si se trouve interrompue, on recherchera le ou les contacts du côté gauche responsables de cette rupture de guidage. Nous pourrons ainsi objectiver le ou les contacts ou plages de contacts qualifiés d'interférences occlusales non travaillantes à l'aide de ruban marqueur fin de $10 \mathrm{ou}$ 20 micromètres.

Interférences travaillantes : de la même manière, des contacts situés du côté droit mastiquant (ou travaillant) sur des portions anatomiques non destinées au guidage (cuspides linguales mandibulaires par exemple) sont susceptibles de perturber le guidage en offrant un obstacle qui va réaliser une rupture de la zone de guidage.

\section{INCIDENCE SUR \\ LE FONCTIONNEMENT DE L'APPAREIL MANDUCATEUR}

Si l'on s'en tient à une analyse mécaniste du fonctionnement de l'appareil manducateur, ces interférences occlusales ne semblent pas très favorables puisqu'à

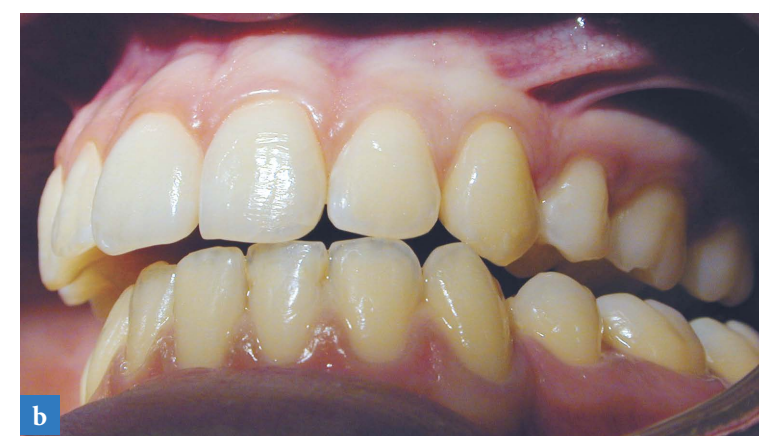

$\triangle$ Fig. 1a et b : Les trajets en latéralité sont le plus souvent guidés par les canines et incisives latérales maxillaires. Cette relation dynamique est le plus souvent rencontrée en l'absence de dysharmonie occlusale.
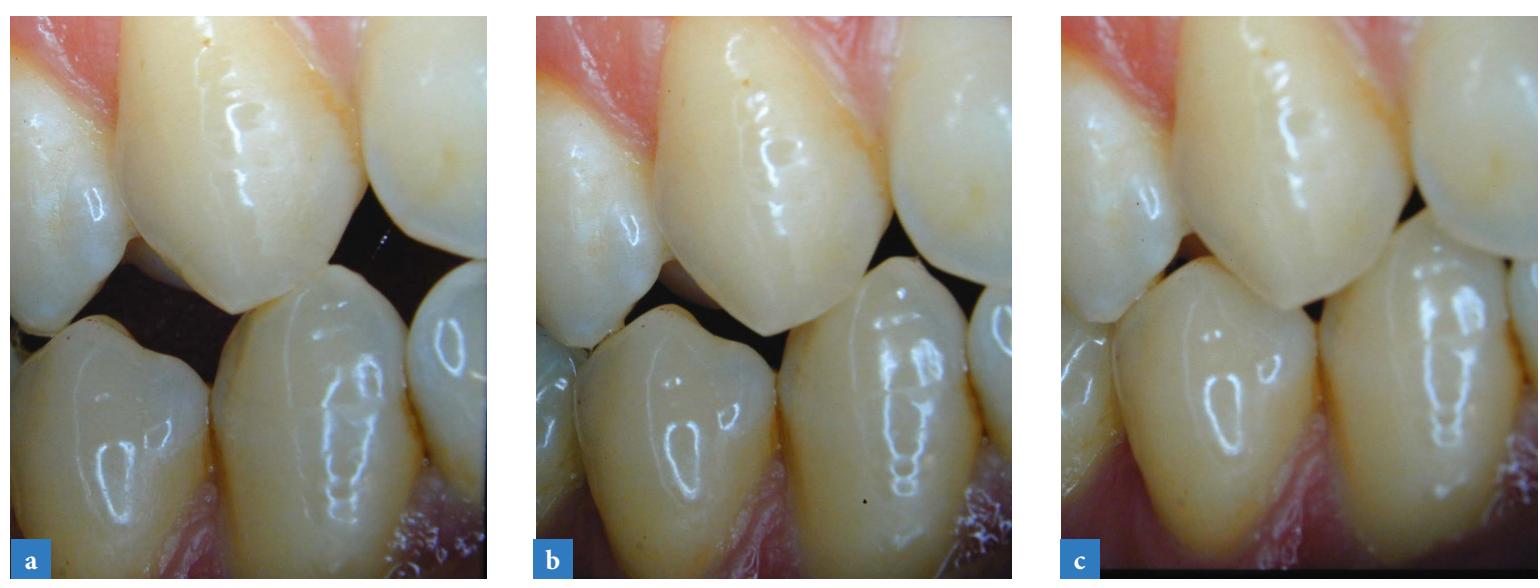

$\triangle$ Fig. 2a, b, c : Le guidage est parfois réparti sur plusieurs dents du côté travaillant incluant canine, prémolaire et parfois molaire. Si le guidage n'est pas interrompu lors d'un mouvement centripète en partant du bout à bout canin, nous ne sommes pas en présence d'interférence non travaillante, même s'il existe simultanément des contacts du côté non travaillant. 
l'approche de l'occlusion d'intercuspidation maximale lors d'un cycle de mastication ou lors de l'incision, la mandibule ne pourra se déplacer facilement du fait de ces obstacles. Indéniablement, cela demandera un effort accru de régulation aux muscles élévateurs, pouvant générer des co-contracion des muscles masticateurs antagonistes (contraction simultanée de muscles élévateurs et abaisseurs). Ces considérations et une lecture " mécaniste « du fonctionnement de l'appareil manducateur ont contribué à souligner l'incidence pathogène de ces interférences occlusales jusque dans les années 90 et au-delà. En fait, la capacité de régulation de l'appareil manducateur est énorme et la majorité des patients s'accommodent très bien de ces anomalies occlusales, "particularité anatomique « selon CM VALENTIN qui, dans une direction donnée de mouvement, seraient susceptibles d'en changer le sens, SI ELLES N'ÉTAIENT PAS ÉVITÉES (Valentin 1996). Les muscles abaisseurs sont capables dans la plupart des situations de réguler les déplacements mandibulaires afin d'éviter les heurts et les obstacles naturels que constituent les interférences occlusales non travaillantes en diduction et en propulsion. Observons toutefois que les interférences potentiellement gênantes se situent à l'approche de l'OIM et prennent place lors de l'accès à l'OIM dans un mouvement centripète lors du cycle masticatoire (Fougeront, 2014). Il est donc intéressant de les dépister lors d'un mouvement guidé centripète du même type en soutenant la mandibule, ce qui mimera un déplacement proche de la réalité lors d'un cycle fonctionnel de mastication.

Toutefois, lorsque les capacités d'adaptation du patient sont dépassées (muscle douloureux, mobilité dentaire, dysfonctionnement de langue, bruxisme), les interférences peuvent compliquer à l'excès le fonctionnement économique des muscles et contribuer à l'épuisement de ceux-ci en constituant un facteur d'entretien des myalgies (Seligman, 1991). Dans ce contexte, les orthèses occlusales trouvent une indication de choix en offrant un schéma occlusal simplifié (contacts généralisés dans les secteurs cuspidés, guidage antérieur reconstitué par une zone de guidage en résine). Ce type d'orthèse pourra être portée quelques semaines et permettra aux muscles de retrouver leur capacité adaptative à la malocclusion que l'on pourra alors qualifier de fonctionnelle.

\section{BRUXISME ET INTERFÉRENCES OCCLUSALES}

Les choses se compliquent en présence de bruxisme, ennemi historique des chirurgiens-dentistes puisqu'il est souvent délétère pour la denture (usures, fractures, migrations). Par là même, le patient qui bruxe en grinçant depuis plusieurs années présente une malocclusion du fait de la perte des éléments de calage et de guidage qui ne remplissent plus leur rôle et laissent place aux contacts exclusifs du côté non mastiquant (les interférences non travaillantes), voire des interférences travaillantes. La malocclusion va évoluer avec les pertes de substances et il sera de plus en plus difficile d'intégrer de nouveaux éléments de dentisterie restauratrice dans ce contexte. En effet le chemin d'accès à l'OIM devient de plus en plus difficile à négocier d'autant que les muscles sont sollicités de façon excessive avec des durées de contacts dentaires bien plus importantes que pour un patient non bruxiste et des forces multipliées par 20 (Rugh, 1988).

La capacité de régulation est très souvent dépassée et les "patients bruxent sur leurs interférences occlusales ». Cette fois les inquiétudes formulées initialement sur le risque d'instabilité occlusale et de dysfonctionnement musculo-squelettiques peuvent être prises en considération. Nous avons vu la complexité de régulation musculaire pouvant entraîner des phénomènes de fatigue, crampes, douleurs, bien que le déclenchement de la myalgie soit rarement le fait du surcroît d'activité lors des contractions, contractures prolongées (Okeson, 1998). D’ailleurs, la grande majorité des patients qui bruxent lors du sommeil et présentent des grincements plus ou moins sévères ne présentent pas de douleur musculaire ni articulaire. Paradoxalement, le bruxisme de sommeil peut être considéré comme un facteur d'entretien des myalgies de l'appareil manducateur (Fleiter, 2015). Les interférences occlusales seront plus la conséquence du bruxisme et certainement pas la cause comme on a pensé à une époque ; les dents restent les victimes.

\section{UTILITÉ DE L'ARTICULATEUR POUR REPÉRER UNE INTERFÉRENCE OCCLUSALE}

Il est impossible de dépister une interférence occlusale sur l'articulateur seul, puisque l'articulateur ne permet pas de réaliser des mouvements fonctionnels. Tout au plus, il peut reproduire des déplacements mandibulaires guidés par les dents antérieures et les dents cuspidées assurant le guidage du côté mastiquant. Cependant l'articulateur, s'il ne peut pas reproduire un cycle de mastication, qui de toute manière possède un caractère aléatoire sauf à l'approche de l'OIM ou les zones de calage et de guidage assurent l'élévation complète de la mandibule en OIM, permettra de visualiser des interférences « possibles » validées en clinique 
puisque le réglage de la pente condylienne de l'articulateur va influencer l'espace interocclusal molaire à l'approche de l'OIM. Ainsi on préférera réduire volontairement la valeur de cette pente condylienne à des valeurs de 25 degrés (alors que la valeur moyenne est proche de 40 degrés) afin de ne pas risquer de générer un espace interocclusal supérieur à la réalité clinique. D’ailleurs, bon nombre d'articulateurs sont préréglés et souvent fixés à 25 degrés de pente condylienne pour ces raisons. L'articulateur ainsi réglé ne risque pas de masquer des interférences occlusales du fait d'un abaissement excessif de la boule condylienne et donc de l'hémi-arcade mandibulaire du côté non travaillant (Chateau, 2005).

Pour résumer, les interférences occlusales sont dépistées à la clinique et peuvent être objectivées sur l'articulateur.

\section{ALORS QUAND INDIQUER UNE HARMONISATION DES CONTACTS OCCLUSAUX ?}

Les équilibrations occlusales soustractives ne sont jamais indiquées dans le but de prendre en charge des ADAM (Algies et Dysfonctionnement de l'Appareil Manducateur) en tous les cas en première intention. Lorsque des interférences occlusales sont observées

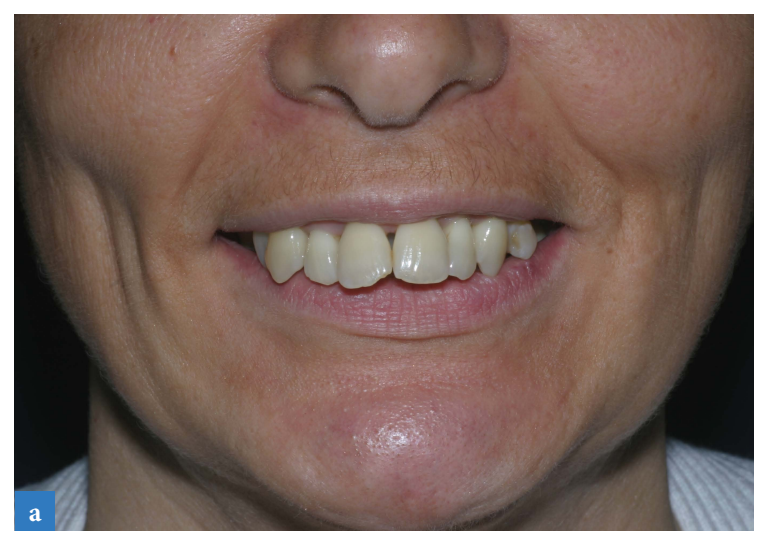

$\triangle$ Fig. 3a, b, c: Patiente de 45 ans, présentant une dysharmonie dento-maxillaire dans un contexte parodontal stabilisé mais affaibli avec dysfonctionnement lingual expliquant les migrations secondaires. sur des dents de sagesse par exemple et qu'une orthèse occlusale à permis de réduire les symptômes douloureux, l'extraction des dents de sagesse constituant l'interférence sera parfois indiquée. De façon exceptionnelle des modifications occlusales soustractives pourront être envisagées lorsque la validation a été conduite par le port d'une orthèse occlusale avec résolution des symptômes. L'orthodontie pourra trouver un intérêt dans ces situations en vue de pérenniser les améliorations initialement observées par la kinésithérapie ou le port d'une orthèse occlusale (Michelotti, 2010 ; Fleiter, 2009)

Attention néanmoins, toute modification occlusale n'est pas anodine et peut parfois être difficile à accepter par le patient du fait de la perte des repères d'OIM. D'autre part cette intervention, si elle n'est pas très bien expliquée en amont, risque d'être ressentie comme une mutilation!

Ceci ne veut pas dire qu'il ne faut jamais la faire en particulier dans le cas où des constructions prothétiques sont envisagées (Chateau, 2005). Ainsi l'équilibration occlusale préprothétique conserve ses lettres de noblesse et facilite grandement l'intégration des constructions. La démarche la plus économique sur le plan biologique reste bien entendu l'orthodontie préprothétique qui permettra de retrouver une situation dento-squelettique stable après évaluation du dysfonctionnement lingual (Fig. 3a à i). Les modifications occlusales envisagées seront conduites sur
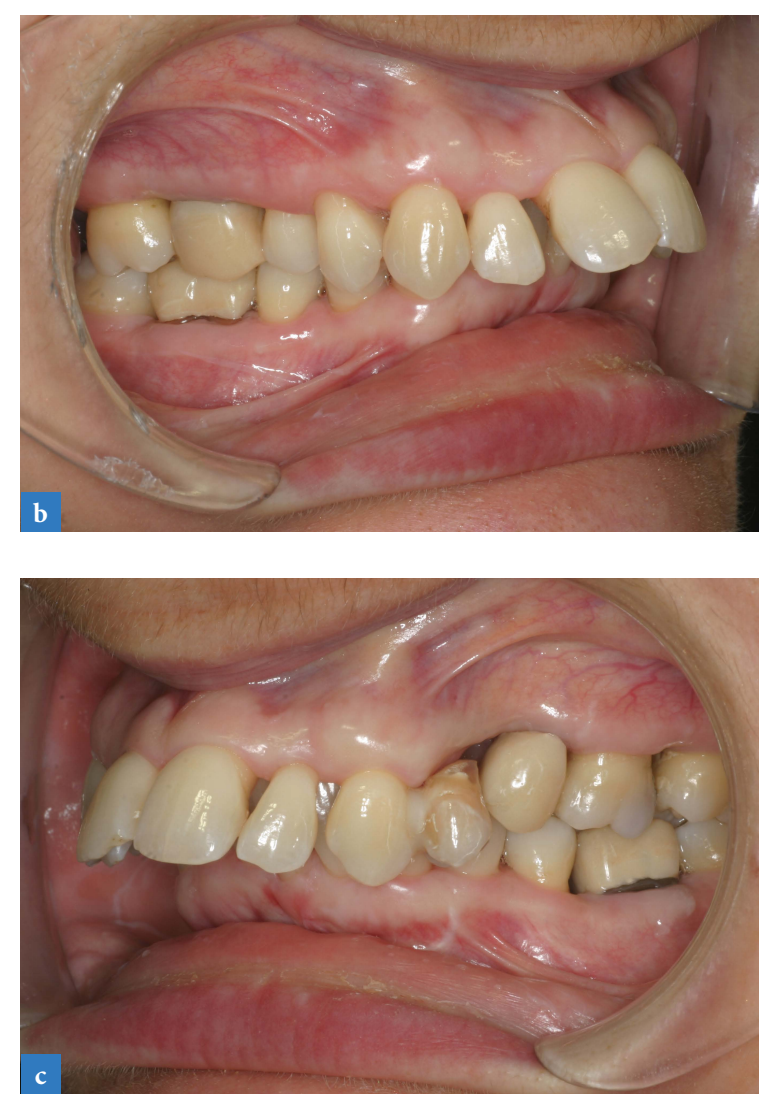

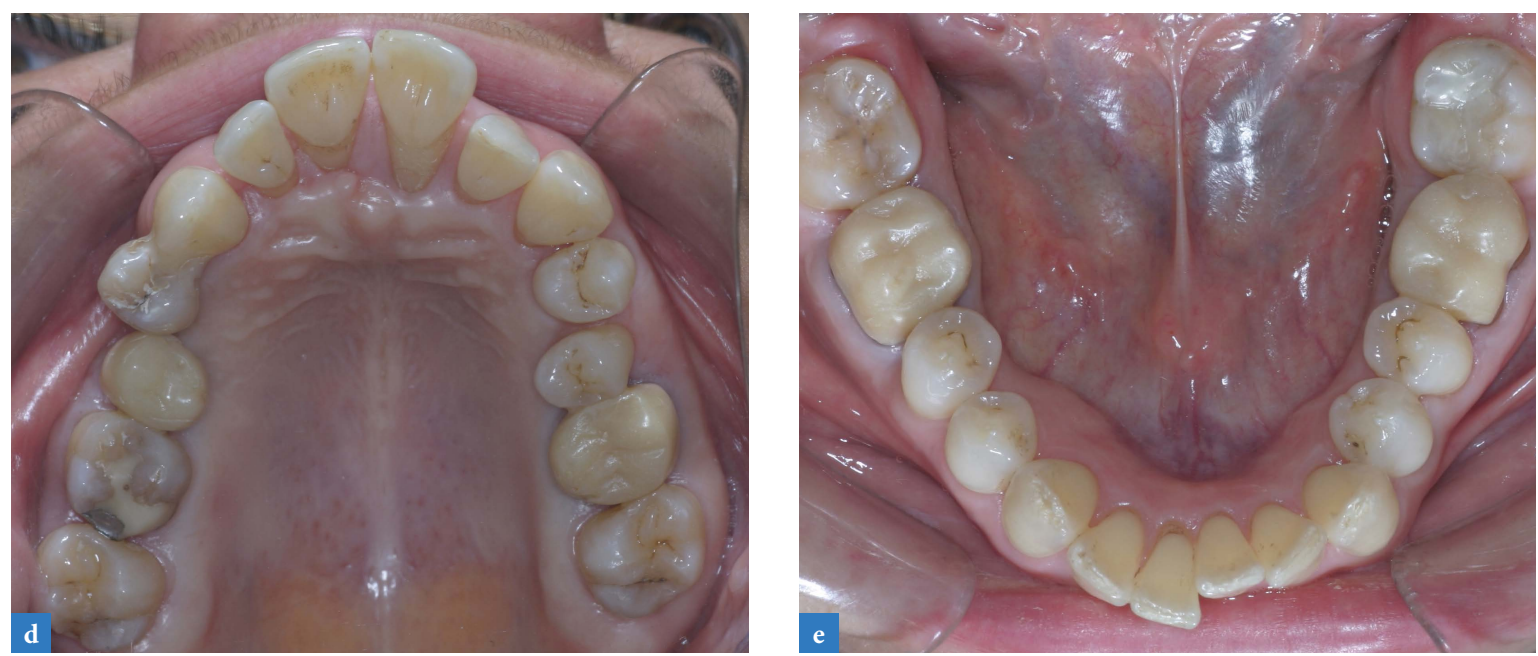

$\triangle$ Fig. 3 d, e : Vue clinique avant traitement orthodontique.

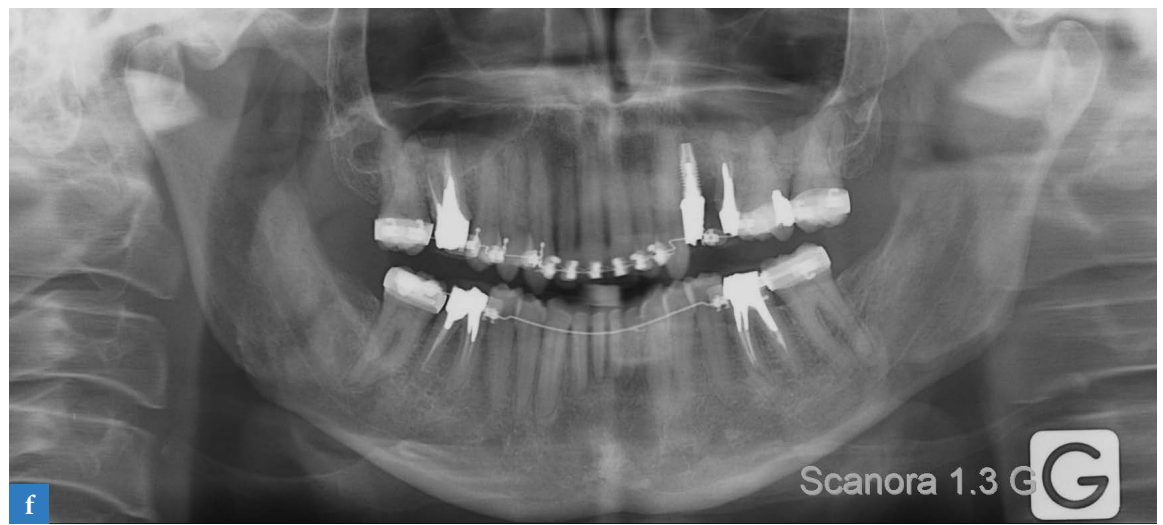

$\checkmark$ Fig. 3f :

Orthopantomogramme

réalisé en cours de traitement orthodontique. On note

l'implant unitaire au niveau

23 , utile comme ancrage orthodontique lingual.
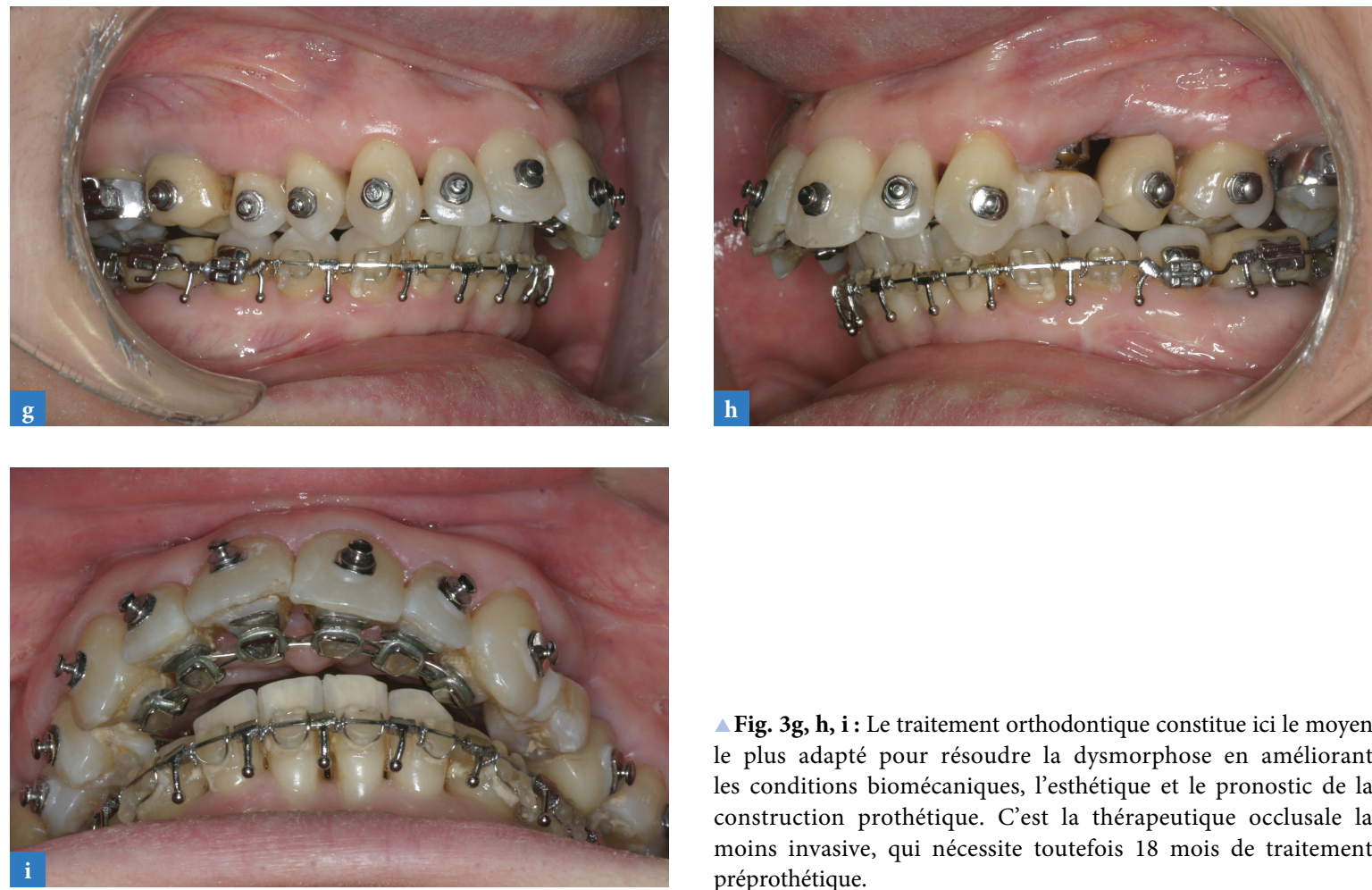

$\Delta$ Fig. $3 g$, h, i : Le traitement orthodontique constitue ici le moyen le plus adapté pour résoudre la dysmorphose en améliorant les conditions biomécaniques, l'esthétique et le pronostic de la construction prothétique. C'est la thérapeutique occlusale la moins invasive, qui nécessite toutefois 18 mois de traitement préprothétique. 
l'articulateur en intégrant le projet en cire ou autre matériau permettant aux praticiens, prothésiste et patient de visualiser et valider le projet dans son ensemble avant toute intervention. Une telle démarche rassurera l'ensemble des protagonistes et évitera des discussions tardives sur le bien fondé de l'équilibration occlusale

\section{ET LA LANGUE ?}

À l'issue d'une équilibration occlusale il n'est pas rare que des patients ne se sentent pas bien dans leur bouche. Ils avouent volontiers que les dents touchent partout mais ils éprouvent des difficultés accrues lors de la déglutition (Belet, 2015 ; Gil, 2015). La dimension verticale d'occlusion n'a pas été diminuée, ce qui est en général le plus souvent le cas dans les techniques d'équilibration (Château, 2005), mais la langue ne trouve plus sa place. Ceci est observé en particulier lorsque l'on tente de retrouver par équilibration des contacts sur les dents antérieures, inexistants auparavant.

Le dysfonctionnement lingual pré-existant constitue, de ce fait, une contre indication à l'équilibration soustractive car celle-ci ne pourra plus s'interposer comme avant entre ou contre les incisives. La rééducation linguale préalable à l'équilibration permettra de déjouer ce piège fréquent, à moins que l'on ne s'accommode d'une absence de guidage antérieur et que l'on puisse reporter ou maintenir les zones de guidage sur des dents plus postérieures (prémolaires par exemple). Le prothésiste et le praticien ne pourront pas, dans ce cas, prétendre à l'abaissement immédiat de la mandibule dès que celle-ci quitte l'OIM, ce qui rendra plus complexe le réglage et l'intégration des éléments de dentisterie restauratrice. L'articulateur dans ce cas sera d'une aide précieuse et on aura bénéfice à programmer cette fois les boîtiers condyliens au plus proche de la pente condylienne du patient.

\section{CONCLUSION}

L'incidence pathogène des interférences occlusales s'avère très discutable, du moins sur l'articulation temporo-mandibulaire et les muscles masticateurs du fait de la capacité adaptative de l'appareil manducateur. Toutefois, les malocclusions sévères constituent des éléments pouvant altérer le pronostic des traitements de dentisterie restauratrice et les thérapeutiques occlusales additives, soustractives ou orthodontiques sont souvent indiquées dans ces situations. Une grande attention sera apportée à la finition des traitements afin d'assurer la plus grande stabilité occlusale en intercuspidation maximale.

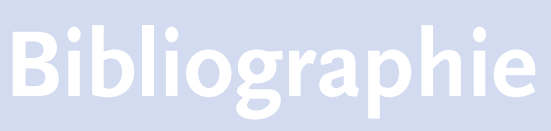

[1] Belet M, Fleiter B, Cazals G. Dysfonctionnement de la langue et myalgies de l'appareil manducateur. Info Dent 2015; 21 : 74-80.

[2] Chateau N, Fleiter B. Conduite de l'équilibration occlusale pré-prothétique Réal Clin 2005; 16:39-51.

[3] Fleiter B. Algies et troubles fonctionnels de l'articulation temporo-mandibulaire. Douleurs : Évaluation, Diagnostic, Traitement 2009;10(3):136-41.

[4] Fleiter B, Fougeront N, de Jaegher P. Troubles musculo-squelettiques de l'appareil manducateur. Ed Quintessence Int. Paris 2015.

[5] Fougeront N, Garnier B, Fleiter B. Motricité, fonctions manducatrices et réflexes de la mâchoire. Med Buccale Chir Buccale 2014;20(3):161-70

[6] Gil H, Fougeront N. Dépister un dysfonctionnement lingual : bilan à l'usage des prescripteurs. Rev Orthop Dento Faciale 2015;49:277-92.

[7] Michelotti A, Iodice G. The role of orthodontics in temporomandibular disorders. J Oral Rehabil 2010;37(6):411-29.

[8] Okeson JP. Management of temporomandibular disorders and occlusion. St Louis : CV Mosby, 1998.
[9] Orthlieb JD. Gnathologie fonctionnelle. Vol 1: Occlusion et restauration prothétique. Paris : Éditions CDP, 2010.

[10] Rugh J, Zarb,G, Mohl N, Carlsson G. Textbook of occlusion. Ed Qintessence Int Chicago 1988.

[11] Seligman DA, Pullinger AG. The role of intercuspal occlusal relationships in temporomandibular disorders: a review. J Craniomandib Disord 1991;5(2):96-106.

[12] Valentin C, Dowek D, Fleiter B. Pertinence et fiabilité de l'examen clinique dans les désordres temporomandibulaires. Réalités Cliniques 1996;7(2):177-96. 